\title{
The therapeutic approach of acute diarrheic disease in pediatric patient
}

\author{
Andreea Ligia Dinca ${ }^{1,2}$, Cristina Oana Marginean ${ }^{1,2}$, Iulia Armean', \\ Vladut Stefanut Sasaran ${ }^{2}$, Lorena Elena Melit ${ }^{1,2}$ \\ ${ }_{1}^{1}$ Pediatrics Clinic 1, Emergency Clinical County Hospital, Tg. Mures, Romania \\ ${ }^{2}$ University of Medicine and Pharmacy, Tg. Mures, Romania
}

\begin{abstract}
Objectives. Approaching acute diarrheal disease (ADD) in pediatric patient, we have set as a primary objective a throughout evaluation of etiology and risk factors and, on the other hand the risk/benefit assessment regarding the justification of antibiotic therapy in the treatment of ADD in pediatric patient.

Material and method. We performed an analytic, descriptive study, in which we included 125 patients admitted in the 1st Pediatrics Clinic of Tg. Mureș during 2016 (January to December), diagnosed with ADD.

Results. The study group comprised 125 patients, out of which a bacterial etiology was noticed in 23 cases (18.4\%). On the other hand, it was observed that 84 patients received antibiotics $(67.2 \%), 110$ patients $(88 \%)$ received symptomatic treatment, and in $60 \%$ of the cases (75 patients), a probiotic was associated. The medium hospitalization length was of 5 days, depending on the presence/absence of risk factors such as fever, inflammatory syndrome, hydro-electrolytical imbalances or acute dehydration syndrome, risk factors that increased the medium hospitalization length with up to 2 days $(p<0.05)$.

Conclusions. Antibiotics must be used rationally and justified in the treatment of ADD, analyzing carefully the risk/benefit ratio. Taking into account the self-limiting character and the favorable prognosis of this disorder, we conclude that we face an abuse of antibiotics in case of pediatric patients diagnosed with ADD.
\end{abstract}

Keywords: acute diarrheic disease, child, antibiotics

\begin{abstract}
Abbreviations
ADD - acute diarrheic disease

IBD - inflammatory Bowel Disease

ADS - acute dehydration syndrome

IgE - immunoglobulins type E

$\mathrm{CRP}-\mathrm{C}$ reactive protein
\end{abstract}

\section{INTRODUCTION}

Gastroenteritis or acute diarrheic disease $(A D D)$ is a non-specific term that describes a series of pathological phenomena localized in gastrointestinal tract, determined by a viral, bacterial or parasitic pathogen. The high morbidity of this pathology (approximately 1.8 billion episodes of $\mathrm{ADD} / \mathrm{year}$, has a major impact in Healthcare regarding both clinical and socio-economical levels (1).

The most frequent etiologies of ADD are represented by viral agents. On the other hand, Salmonella spp., Shigella spp. and Campylobacter spp. represent the most frequent bacterial cause of ADD among pediatric patients $(2,3)$.

The treatment principles of ADD include: oral rehydration therapy, enteral feeding, hygienic and dietetic treatment, but also adjuvant therapies, such as probiotics. Etiological treatment should be used in all cases where the etiology is identified. The use of probiotics in the prophylaxis and treatment of ADD is generally of great benefit. In addition to re-equilibration of the intestinal flora, probiotics also play an important role in stimulating host's immunity - by "down-regulation" of pro-inflammatory cytokines and "up-regulation" of anti-inflamma- 
tory cytokines phenomena (1). The early antibiotics treatment in selected cases of ADD in child, can lead to diminishing the length and severity of the disease, but also to the prevention of associated complications. Their use on a wide scale, and sometimes without indication, led to the development of bacterial resistance. Most times, the decision of antibiotic initiation empirical, without a solid medical justification (4).

\section{MATERIAL AND METHOD}

We performed a descriptive, analytical, retrospective study, in which were included 125 pediatric patients, admitted to the $1^{\text {st }}$ Pediatrics Clinic of Tîrgu Mures during 2016 (from January-December). Our main objective was to perform a detailed assessment of the etiology and risk factors of ADD, but also to assess the risk/benefit ratio regarding the judgement in antibiotics treatment.

The inclusion criteria consisted in: patients admitted to $1^{\text {st }}$ Pediatrics Clinic of Tîrgu Mures between January the $1^{\text {st }}$ and December the $31^{\text {st }} 2016$, aged from 0 and 18 years who presented for ADD symptoms, or patients with the onset of ADD during admission. The exclusion criteria consisted in: presence of chronic diarrhea (with a length above 14 days), incomplete clinical or paraclinical data. The data were analyzed and processed with SPSS statistical software (Mann Whitney and Chisquared tests). The confidence interval was settled to a value of $95 \%$, and the results were considered significantly statistical for a value of significance threshold " $p$ " $<0.05$.

\section{RESULTS}

The incidence of ADD in the $1^{\text {st }}$ Pediatrics Clinic was of $7.3 \%$ (125 cases) out of all admissions $(1,702)$ in year 2016 .

The study group comprised 125 patients, among which 71 (56.8\%) were males, and 54 (43.2\%) were females. The patients were divided on age groups as it follows: infants - 60 patients (48\%), small children, aged between 1 and 3 years old -37 (29.6\%), and children older than 3 years -28 patients (22.4\%). Therefore, we identified the highest incidence of ADD during small infancy period.

We found a $71.2 \%$ percentage (89) of cases manifested during the cold season, and $28.8 \%$ (36 cases) during the hot season. As for the etiology of ADD, we identified a bacterial etiology in 23 cases (18.4\%), meanwhile viral gastroenteritis accounted for 53 of the cases (42.4\%). The remaining of the cases were due to other etiologies (Table 1).

Rotavirus infection was identified in 13 patients $(10.4 \%)$ of all ADD cases. Among other viral agents, Adenoviruses were also tested. Regarding the bacterial intestinal infections, the stool culture tested the following species: Campylobacter, Escherichia coli (E.coli) enteropathogenic, Yersinia, Shigella, Salmonella. Therefore, the infectious agents were identified in 4 cases, out of which 2 patients with E.coli enteropathogenic, one case with Salmonella spp. A case of Clostridium difficile infection was identified in a 2 and a half yearold patient, who was diagnosed based on a positive GDH (glutamate dehydrogenase) test, and subsequently the evidence of toxins A and B from feces. Parasitological stool examination was positive in 3 cases. Stool culture provides information on etiology in rare cases. Bacterial origin was considered in ADD cases associated with a profoundly altered general condition, hyperpyrexia, leukocytosis with neutrophilia, the presence of leukocytes and pathological elements in feces, as well as the presence of a severe inflammatory syndrome (modified CRP > $10 \mathrm{mg} / \mathrm{dl}$ ). Dysbiosis was incriminated as the etiologic factor of the BDA in the specified cases in which the dysbiosis phenomenon was described based on the result of stool culture, or in patients who had undergone prolonged antibiotic treatment and developed a characteristic ADD.

TABLE 1. The etiology of ADD

\begin{tabular}{|l|c|c|c|}
\hline ETIOLOGY & Identified agent & No case & $\begin{array}{c}\text { Percentage } \\
\text { ADD }\end{array}$ \\
\hline BACTERIAL & E.coli enteropathogenic & $\mathbf{2}$ & $1.6 \%$ \\
\hline 23 cases & Clostridium difficile & $\mathbf{1}$ & $0.8 \%$ \\
\hline (18.4\%) & Salmonella spp. & $\mathbf{1}$ & $0.8 \%$ \\
\hline VIRAL & Other & $\mathbf{1 9}$ & $15.2 \%$ \\
\hline 53 cases & Rotavirus & $\mathbf{1 3}$ & $10.4 \%$ \\
\hline $\begin{array}{l}\text { (42.4\%) } \\
\text { PARASITIC } \\
\text { (4 cases) }\end{array}$ & Other viral e ologies & $\mathbf{1 9}$ & $0.8 \%$ \\
\hline $\begin{array}{l}\text { ALLERGIC } \\
\text { (16 cases) }\end{array}$ & Oxiuriasis & $\mathbf{3}$ & $31.2 \%$ \\
\hline $\begin{array}{l}\text { IBD } \\
\text { (8 cases) }\end{array}$ & Specific Ig E & $\mathbf{9}$ & $7.4 \%$ \\
\hline $\begin{array}{l}\text { DISBIOSIS } \\
\text { (21 cases) }\end{array}$ & - & $\mathbf{8}$ & $6.4 \%$ \\
\hline IDIOPATIC & - & $\mathbf{2 1}$ & $16.8 \%$ \\
\hline TOTAL: & - & $\mathbf{2 7}$ & $21.6 \%$ \\
\hline
\end{tabular}

Among the symptoms frequently identified in our study were: vomiting (56 patients) and fever (64 patients). Paraclinically, we noticed the presence of inflammatory syndrome proved by positive 
C-reactive protein (CRP) in 55 patients (44\%), of hydro-electrolytical imbalances in 49 cases (39.2\%), and of acute dehydration syndrome (ADS) in 82 of the cases $(65.6 \%)$.

The administered treatment was compounded of antibiotics, symptomatic and probiotics. We noticed that 84 patients received antibiotic treatment $(67.2 \%)$, while 110 patients $(88 \%)$ required symptomatic treatment. Symptomatic were used: antiemetic medications such as Metoclopramide, antidiarrheic agents such as diosmectitis and racecadotril, and drotaverine by vein as an antispasmodic. In $60 \%$ of cases (75), probiotic preparations were associated within treatment.

The medium hospitalization length of the patients was 5 days. We identified a significantly statistical association between fever $(p=0.0164)$, the positive inflammatory syndrome $(\mathrm{p}=0.0124)$, the presence of hydro-electrolytical imbalances $(\mathrm{p}=$ $0.0064)$, the acute dehydration syndrome $(\mathrm{p}=$ $0.0034)$ and the increase of medium hospitalization length (Table 2).

TABLE 2. The influence of risk on the hospitalization length

\begin{tabular}{|l|c|c|c|}
\hline MODIFICATIONS & YES & NO & ' $p$ ' VALUE \\
\hline & \multicolumn{3}{|c|}{ The number of hospitalization days } \\
\hline FEVER & 5 & 4 & 0.0164 \\
\hline Positive CRP & 6 & 4 & 0.0124 \\
\hline $\begin{array}{l}\text { HYDRO-ELECTROLYTICAL } \\
\text { IMBALANCES }\end{array}$ & 5.5 & 4 & 0.0064 \\
\hline ADS & 5 & 3 & 0.0034 \\
\hline
\end{tabular}

The gender has no statistical significant influence on the development of ADD $(p=0.66)$. Despite the fact that $48 \%$ of the patients included in our study were infants, we did not find any significantly statistical associations between the studied age groups and the development of ADD. We also assessed the impact of seasons on the development of ADD, but we did not identify any significant associations $(\mathrm{p}=0.48)$.

\section{DISCUSSIONS}

ADD represents one of the main cause of morbidity, and even mortality among pediatric patients. The present study analyzed 125 cases of ADD regarding the incidence on genders and age groups, etiology, risk factors and therapeutic approach.

Specialty studies indicate increased morbidity due to ADD in the young child, and especially in the infant (5). In the current study, the highest incidence of ADD was found in infants $(60$ subjects $48 \%$ ) and approximately equal proportions with the other age groups $(29.6 \%$ for young children and $22.4 \%$ for over 3 years). Infancy is a special category in pediatric pathology due to high susceptibility to dehydration, nonspecific symptomatology that may accompany ADD and increased risk of complications. Contrary to expectations, the study did not reveal a statistically significant association between the primary diag-nosis of ADD and the age groups studied $(\mathrm{p}=0.12)$.

The season influence on the development of ADD is frequently incriminated in the literature. Usually, it is associated with rotavirus infections and it describes a predilection for the cold season (November-April) (6). The seasonality of ADD is in a progressive decline due to the anti-rotavirus immunization regimens implemented worldwide $(6,7)$. The present study pointed partially the seasonality character of ADD, thus $71.2 \%$ of the cases were diagnosed during the cold season, in comparison to $28.8 \%$ cases that were admitted during the hot season. Nevertheless, we did not find a significantly statistical correlation regarding the role of seasonality in the development of ADD as a main diagnosis $(\mathrm{p}=0.48)$.

The main etiological agents were of viral origin (42.2\%) and approximately a quarter were infection caused by rotavirus. These results followed the pattern described by other studies from the literature, pointing a high incidence of rotavirus infections in viral $\operatorname{ADD}(8,9)$.

Among the 23 patients group with bacterial ADD, we identified the etiological agent in only 4 cases. We can state that the diagnostic method owns high specificity, but a low detection rate for the etiological agent in bacterial ADD. One of the limitations of the study refers to the impossibility of testing several bacterial species through stool culture, and consequently to the lack of an etiological diagnosis regarding ADD of bacterial origin. Also, a more comprehensive testing of viral species could have provided a significant contribution to the scientific quality of the study.

The clinical picture in ADD can be non-specific, being dominated by diarrhea, with or without other gastrointestinal/systemic symptoms. The severity of the disease depends mostly on the degree of patient's dehydration and it is correlated with the hospitalization length, the complexity of the treatment and the moment of therapy initiation.

The fever is not a risk factor for the development of ADD, and is a non-specific symptom. Nevertheless, the studies within the medical area associate frequently fever with an unfavorable prognosis in $\operatorname{ADD}(10,11)$. We can state the same thing about 
the presence of inflammatory syndrome (positive CRP), of hydro-electrolytical imbalances and ADS. We did not identify in the study pathognomonic modifications regarding the paraclinical picture in ADD.

The medium hospitalization length for the patients included in our study was of 5 days. The hospitalization period presents negative effects regarding both the socio-economical level, and the quality of patient's and care-givers' lives. Therefore, ADD is a pathology with a significant socio-economic impact (12).

We noticed the fact that the presence of fever, positivity of CRP, the presence of hydro-electrolytical imbalances or ADS present a significant impact on the prolonging hospitalization length. This fact proves the multifactorial character of ADD and the importance of adequate management of this condition.

According to specialized guidelines (11), antibiotics are not primarily used in ADD management and are reserved for cases with specific indication. From the treatment point of view, in the present study, an important use of antibiotic therapy $(67.2 \%)$ is noted in the therapeutic approach of the ADD, in contradiction with the $18.4 \%$ of cases identified as being representative of bacterial etiology. The main types of antibiotics used were Ampicillin, cephalosporins of the $2^{\text {nd }}$ and $3^{\text {rd }}$ generation (Cefuroxime, Ceftriaxone), and in selected cases (sepsis) broad-spectrum antibiotics such as carbapenems were used.

The increased percentage of the patients treated with anti-infectious agents, suggests an unjustified choice in antibiotics utility. We underline the importance of antibiotics limitation due to the risk of high bacterial resistance. In addition, the abuse or irrational use of antibiotics, can lead to the development of dysbiosis, and subsequent bowel transit disorders.

An aspect that is intensely studied nowadays, is the association of probiotics in functional bowel disorders. These compounds fulfill essential roles in re-establishing the balance of intestinal flora, in the intestinal humoral immunity and in regulating bowel movements (12). This study indicates the use of probiotics in $60 \%$ of cases, which is justified due to the increased percentage of patients treated with anti-infectious drugs.

\section{CONCLUSIONS}

Rotavirus is the most common etiological agent in viral gastroenteritis, and therefore we support anti-rotavirus vaccination in order to reduce associated morbidity. The physician should be aware of the risk factors associated with a prolonged hospitalization period in case of ADD, such as fever, presence of positive CRP or hydro-electrolytical imbalances. Antibiotherapy should be rationally and reasonably used in treating $\mathrm{ADD}$, by carefully analyzing the risk/benefit ratio concerning the patient's outcome.

Therefore, taking into account the self-limiting character and the favorable prognosis of the disease, we conclude that we are facing an antibiotic abuse in pediatric patients with ADD. The clinician should emphasize more on the symptomatic treatment at the expense of the etiology, since antibiotics have little or no significant impact on the outcome of the disease.

\section{Conflict of interest: none declared Financial support: none declared}

\section{REFERENCES}

1. Bhutta Z.A. Acute gastroenteritis in children. In: Kliegman R.M., Stanton B.F., St. Geme III J.W. et al. Nelson's Textbook of Pediatrics. 20th ed. Philadelphia: Elsevier; 2016:1854-1875.

2. Glass R.I., Parashar U.D., Estes M.K. Norovirus gastroenteritis. N Engl J Med. 2009; 361:1776-1784.

3. DuPont H.L. Bacterial diarrhea. N Engl J Med. 2009; 361:1560-1569.

4. Bhutta Z.A., Nelson E.A., Lee W.S. et al. Recent advances and evidence gaps in persistent diarrhea. J Pediatr Gastroenterol Nutr. 2008;47:260-265.

5. Lanata C.F., Fischer-Walker C.L., Olascoaga A.C. et al. Global causes of diarrheal disease mortality in children, 5 years of age: A systematic review. PloS One.2013, 8 (9):e72788.

6. Tate J.E., Panozzo C.A., Payne D.C. et al. Decline and change in seasonality of US rotavirus activity after the introduction of rotavirus vaccine. Pediatrics. 2009; 124(2):465-471.

7. Fagundes-Neto U. Persistent diarrhea: Still a serious public health problem in developing countries. Curr Gastroenterol Rep. 2013; 15(9):1-6.

8. Glass R.I., Parashar U.D., Estes M.K. Norovirus gastroenteritis. N Engl J Med. 2009; 361:1776-1784.
9. Rendi-Wagner P., Kundi M., Mikolasek A. et al. Active hospitalbased surveillance of rotavirus diarrhea in Austrian children, period 1997 to 2003. Wien Klin Wochenschr. 2006; 118(9-10):280-285.

10. Streit J.M., Jones R.N., Toleman M.A. et al. Prevalence and antimicrobial susceptibility patterns among gastroenteritis-causing pathogens recovered in Europe and Latin America and Salmonella isolates recovered from bloodstream infections in North America and Latin America: Report from the SENTRY Antimicrobial Surveillance Program (2003). Int J Antimicrob Agents. 2006; May, 27(5):367-375.

11. Guarino A., Albano F., Ashkenazi S. et al. European Society for Pediatric Gastroenterology, Hepatology and Nutrition/European Society for Pediatric Infectious Diseases Evidence-based Guidelines for the Management of Acute Gastroenteritis in Children in Europe. JPGN. 2008; 46:S81-S122.

12. Freedman S.B., Ali S., Oleszczuk M. et al. Treatment of acute gastroenteritis in children: An overview of systematic reviews of interventions commonly used in developed countries. Evid Based Child Health. 2013; 8(4):1123-1137. 